Sir,

\section{Delayed recurrence of ciliary body melanoma following local resection}

Primary uveal melanoma may be treated by a variety of techniques including local resection, brachytherapy, proton beam therapy, transpupillary thermotherapy, and enucleation. ${ }^{1}$ Demonstration of long-term cure following treatment requires life-long follow-up to exclude local recurrence. A case of very delayed local recurrence following local resection of a ciliary body melanoma is reported.

\section{Case report}

A 61-year-old man presented in September 1977 with a ciliary body melanoma in his left eye. Visual acuity was hand movements. The ciliary body melanoma extended circumferentially for 4 clock hours in the temporal periphery with limited posterior extension. There was a total exudative retinal detachment.

Local resection of the ciliary body melanoma was performed. A partial thickness scleral flap was created to expose the tumour that was dissected from the underlying tissues and removed. A small retinal break occurred at the site of dissection, which was treated with a scleral buckle. No adjunctive therapy was performed for the tumour. Histopathology demonstrated a malignant melanoma composed of a mixture of spindle and epithelioid cells with scanty mitoses. There was no evidence of scleral invasion.

The eye made good progress postoperatively with complete retinal reattachment. Visual acuity improved to $1 / 60$ with useful peripheral visual field. A slightly raised pigmented area was observed in the dissection base 2 months postoperatively, which did not change in appearance during subsequent follow-up (Figure 1). It was therefore considered to represent retinal pigment epithelial hyperplasia rather than residual melanoma. Subsequently, the patient remained under annual review.

At review in January 1996, a solid ciliary body and choroidal mass was present in the temporal periphery of the left eye, which was $11 \mathrm{~mm}$ thick on A-scan ultrasonography (Figure 2). The patient initially declined further intervention, but an exenteration was subsequently performed in July 1998 because of suspected local extraocular spread. Histopathology confirmed a recurrent malignant melanoma, with limited extraocular spread adjacent to the lateral rectus attachment. This extended into the anterior subconjunctival space, but was completely excised. The socket healed well with no new evidence of recurrent tumour to date.

\section{Comment}

Local resection of ciliary body and choroidal melanomas enables preservation of the globe with good potential for vision. Patient selection is important for the success of local resection. Current indications include localised bulky tumours greater than $5 \mathrm{~mm}$ thickness and nasally located tumours more than one disc diameter from the optic disc where vision needs to be conserved. ${ }^{1,2}$

However, the technique of local resection is technically demanding and requires hypotensive anaesthesia. Complications of this surgery include incomplete tumour resection, retinal detachment, vitreous haemorrhage, choroidal neovascularisation in the surgical coloboma,

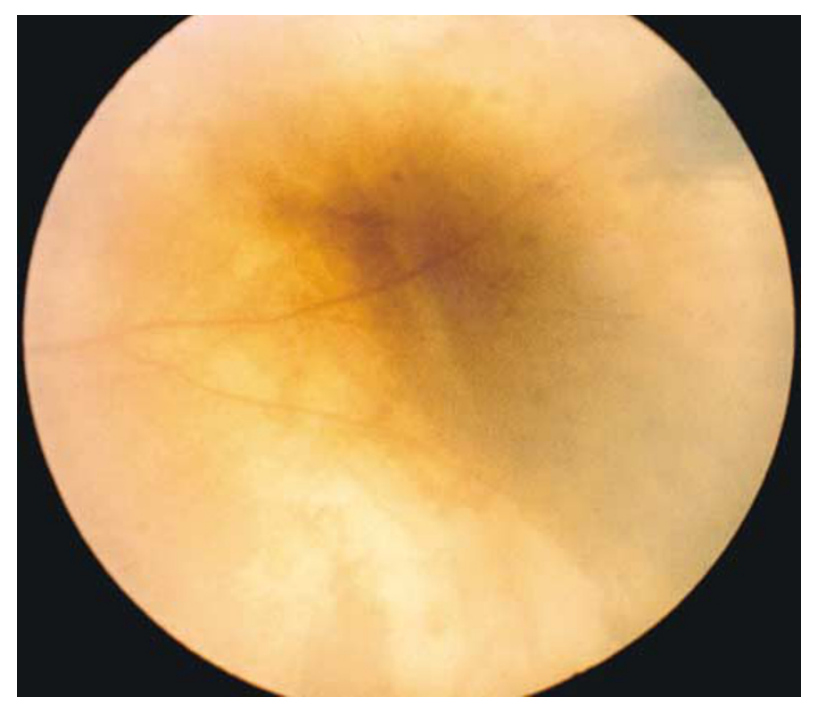

Figure 1 Fundus photograph taken 2 years after local resection showing a slightly raised pigmented area in the dissection base.

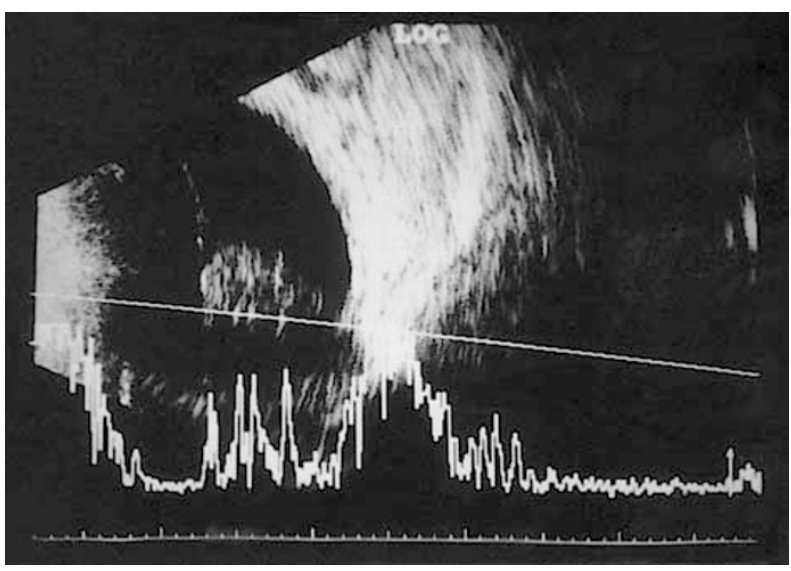

Figure 2 B-scan ultrasonography showing a recurrent ciliary body and choroidal melanoma in the temporal periphery of the left eye. The superimposed A-scan trace demonstrates a tumour thickness of $11 \mathrm{~mm}$. 
cystoid macular oedema, cataract, and phthisis (if more than 2 to 3 clock hours of ciliary body is excised). ${ }^{3,4}$

Local recurrence typically occurs within months to a few years after resection of ciliary body or choroidal melanomas. In one case series, all local recurrences occurred within 7 years of surgery. ${ }^{4}$ There has been one report of local recurrence at 8.5 years after surgery. ${ }^{5}$ In our case, local recurrence occurred 18 years after surgery, which is the longest interval reported to the best of our knowledge. The pathogenesis of local recurrence at such a long interval is probably due to the growth of microscopic deposits of the original tumour that had been controlled by the host-immune response. ${ }^{6}$

Delayed local recurrence has also been reported after the treatment of choroidal melanomas by argon laser photocoagulation (8 years $)^{7}$ and xenon photocoagulation (13 years). ${ }^{8}$ Orbital recurrence has been reported at an interval of 20 years following enucleation. ${ }^{9}$ The occurrence of delayed local recurrence following newer treatment modalities such as proton beam therapy and transpupillary thermotherapy remains to be determined. This case report demonstrates the need for life-long follow-up after treatment of uveal melanomas to exclude local recurrence.

\section{References}

1 Damato BE. Ocular Tumours-Diagnosis and Treatment. Butterworth- Heinemann: Oxford, 2000.

2 Damato BE, Paul J, Foulds WS. Predictive factors of visual outcome after local resection of choroidal melanoma. $\mathrm{Br} J$ Ophthalmol 1993; 77: 616-623.

3 Damato BE, Foulds WS. Surgical resection of choroidal melanoma. In: Ryan SJ (ed). Retina, 2nd ed., Vol. 1. Mosby: St Louis, 1994, pp 803-804.

4 Damato BE, Paul J, Foulds WS. Risk factors for residual and recurrent uveal melanoma after trans-scleral local resection. Br J Ophthalmol 1996; 80: 102-108.

5 Shields JA, Shields CL, Shah P, Sivalingham V. Partial lamellar sclerouvectomy for ciliary body and choroidal tumors. Ophthalmology 1991; 98: 971-983.

6 Tarin D. Cancer metastasis. In: Peckham M, Pinedo H, Veronesi U (ed) Oxford textbook of oncology, Vol. 1. Oxford university press: Oxford, 1995, pp 121-122.

7 Brancato R, Menchini U, Pece A. Enucleation after argon laser photocoagulation for choroidal melanoma. Ann Ophthalmol 1988; 20: 296-298.

8 Akduman L, Olk RJ, Green K. Local recurrence of choroidal melanoma 13 years after successful xenon arc photoablation. Retina 2000; 20: 212-213.

9 Shields JA, Augsberger JJ, Dougherty MJ. Orbital recurrence of choroidal melanoma 20 years after enucleation. $A m \mathrm{~J}$ Ophthalmol 1984; 97: 767-770.

VA Shanmuganathan ${ }^{1}$ and DK Newman²

${ }^{1}$ University of Nottingham, Nottingham, UK

${ }^{2}$ Addenbrookes Hospital, Cambridge, UK
Correspondence: VA Shanmuganathan

Research Fellow

Division of Ophthalmology and Visual Sciences

B Floor, Eye, ENT Block

Queens Medical Centre

Nottingham NG7 2UH, UK

Tel: +441159709796

Fax: + 441159709963

E-mail: vijay_shan2000@yahoo.co.uk

Eye (2004) 18, 755-756. doi:10.1038/sj.eye.6700753

Published online 16 January 2004

Sir,

Mobile phone interference in the ophthalmology department

Communication is greatly facilitated by mobile phones (cell phones) and their use is now commonplace among doctors. The current restrictive policy in ophthalmic departments seems to be unjustified given the absence of evidence. There is no published work relating to such departments or their equipment, and the studies available suggest that there is minimal risk to most hospital equipment.

Mobile networks operate by receiving and transmitting their signals via relay stations at carrier frequencies between 900 and $1800 \mathrm{MHz}$. Electromagnetic interference occurs where the mobile, which is an intentional radio transmitter, causes an electronic piece of equipment to act as an unintentional radio receiver. Global System for Mobile communication (GSM) employs a cellular structure in which a base station is used to provide access over a narrow bandwidth. The telephone senses how close it is to the cellular network antenna and increases or decreases its own energy in order to adapt to its environment (adaptive energy), altering the absolute degree interference of individual telephones in any one place. Interference is highest at the initial stages of an outgoing or incoming call.

The issue of mobile phone interference in hospitals first came to attention in the United Kingdom in 1994 when the UK Medical Devices Agency (MDA) issued a safety notice that prompted a detailed study published in 1997. ${ }^{1}$ Only $4 \%$ of 178 devices examined suffered interference from mobile phones, with less than $0.1 \%$ showing serious effects. They recommended that phones should be switched off in areas where sensitive devices may be used (including operating theatres). None of the devices tested are routinely used in the ophthalmology setting. Irnich and Tobisch ${ }^{2}$ tested more than 220 electronic medical devices in a hospital environment 